\title{
Possible explanations for failures to detect cold fusion
}

\author{
Rainer W. Kühne \\ Lechstrasse 63, W-3300 Brunswick, Germany
}

Received 14 May 1991; revised manuscript received 12 August 1991; accepted for publication I 4 August 1991 Communicated by J.P. Vigier

In a series of experiments Jones et al. have detected neutron excesses having indicated some fusion processes within deuterated metals. However, most efforts to reproduce their experiments have failed. Possible sources of failure of later experiments are discussed in this paper.

Jones et al. claim to have detected neutrons which might have originated from $\mathrm{d}-\mathrm{d}$ fusion at Brigham Young University [1-9], in the Gran Sasso Laboratory [10-12] and at Los Alamos National Laboratory [13-15]. Several authors have tried to explain these observations conventionally. Statistical insignificance was claimed [16], statistical and systematical biases were suggested [17], variations of the cosmic neutron background were considered $[18,19]$, bursts of solar flare neutrons were suggested [20] and cosmic muon catalyzed fusion was proposed $[21,22]$. However, all these explanations can now be excluded $[23,24]$.

Several authors assume that microscopical hot fusion (MHF) could be the source of the neutron excesses above [13-15,23-36]. According to this idea deuterons accumulate around impurities and dislocations in the metal lattice, where these accumulations can grow to bubbles. If there are off-equilibrium conditions these bubbles can form cracks. Simultaneously, there can arise high electric fields which will yield so much energy that the deuterons are able to fuse.

Several arguments support the idea of MHF [24].

(1) Menlove et al. [13] have detected acoustical emissions which have indicated the formation of cracks.

(2) According to the idea of MHF each $\mathrm{cm}^{3}$ of deuterated metal should be able to emit about $10^{4}$ neutrons [28]. This agrees with the observations of Jones et al., Bertin et al. and Menlove et al.
(3) MHF demands that bubbles can arise only if there exist dislocations and impurities. Indeed Menlove et al. have not observed any neutrons where they have cleaned their titanium too well [15].

(4) According to MHF large cracks are able to release very short bursts of neutrons. Dozens of very short neutron bursts have been detected by Menlove et al. [13].

(5) The experimentalists have found out that their bursts have preferentially arisen at temperatures -100 to $0^{\circ} \mathrm{C}[13-15]$. Exactly at these temperatures the crack growth rate has its maximum [15].

However, before one states that MHF could have been the source for the excess neutrons one should explain why so many experiments have failed to reproduce the results above. We will list four possible sources of failure.

(1) MHF occurs only if there are non-stationary conditions, i.e. temperature and/or applied current have to be varied [28]. Note also that Jones has written, "A characteristic feature of our experiments is the generation of non-equilibrium conditions in metal-deuterium systems. We accomplish this, for example, by changing temperatures in $\mathrm{D}_{2}$-gas loaded cells and by pulsing voltages in electrolytic cells." [15]. During a lot of those experiments which have failed to detect excess fusion products neither temperature nor applied current have been varied.

(2) Jones et al. were stimulated to perform their experiments by reports upon high ${ }^{3} \mathrm{He} /{ }^{4} \mathrm{He}$ ratios in solids, liquids and gases associated with areas of high 
heat flow [37-39], high ${ }^{3} \mathrm{He}$ concentrations in metals [40] and an excess heat of Jupiter [41]. These reports and their studies in muon catalyzed fusion [42-46] led Jones et al. to the idea of screening and squeezing effects on $\mathrm{D}_{2}$ molecules within metals $[1,47]$. This idea has been refuted [48-69]. But we have to consider that this idea led Jones et al. [1] suggest to perform high energy ion implantation experiments. However, these experiments are not appropriate to test MHF, because high energy deuterons cannot build any large bubbles. And even if they could the implanted $\mathrm{keV}$ deuterons would yield such a large fusion neutron background that neutrons originating from MHF would be overlooked.

(3) The experiments have to be sensitive enough to test the claimed neutron production rates. For electrolysis cells the claimed rates are $10^{-23}-10^{-24}$ $d-d^{-1} s^{-1}$ (experiments of Jones et al. [1,3-8] and Bertin et al. $[10,11])$. By assuming MHF at deuteron energies of $10 \mathrm{keV}$ the corresponding heat output is $10^{-4} \mathrm{~W} \mathrm{~g}^{-1}$ [24]. For gas loaded cells the claimed rate is $6 \times 10^{-26} \mathrm{~d}-\mathrm{d}^{-1} \mathrm{~s}^{-1}$ (experiment of Menlove et al.; as they were not able to reproduce

Table 1

Electrolysis experiments.

\begin{tabular}{|c|c|c|c|c|}
\hline First author & $\begin{array}{l}\text { Sensitivity } \\
\left(10^{-24} d-d^{-1} s^{-1}\right)\end{array}$ & $\begin{array}{l}\text { Current } \\
\left(\mathrm{mA} / \mathrm{cm}^{2}\right)\end{array}$ & Material & References \\
\hline Armstrong & $?$ & 100 & $\mathrm{Pd}$ & {$[73,74]$} \\
\hline Gai & 0.2 & 200 & $\mathrm{Pd} / \mathrm{Ti}$ & {$[19,75]$} \\
\hline Lewis & 1.5 & 64 & $\mathrm{Pd}$ & {$[76-78]$} \\
\hline Williams ${ }^{a)}$ & 0.5 & $14-530$ & $\mathrm{Pd} / \mathrm{Ti}$ & {$[79,80]$} \\
\hline Salamon a) & 200 & $?$ & $\mathrm{Pd}$ & {$[81,82]$} \\
\hline Kashy & 0.6 & $2-12$ & $\mathrm{Pd}$ & [71] \\
\hline Broer ${ }^{a l}$ & 2.2 & $63-70$ & $\mathrm{Pd}$ & {$[83,84]$} \\
\hline Deakin & 32000 & 300 & $\mathrm{Pd}$ & {$[85]$} \\
\hline Rehm & 40 & $10-650$ & $P d$ & {$[86]$} \\
\hline Southon & 10 & 1000 & $\mathrm{Ti}$ & {$[87]$} \\
\hline Ziegler & 0.2 & 150 & $\mathrm{Pd}$ & {$[88,89]$} \\
\hline Price & 0.06 & 1 & $\mathrm{Pd} / \mathrm{Ti}$ & {$[90]$} \\
\hline Briand $\left.{ }^{b}\right)$ & 100 & 3.3 & $\mathrm{Ti}$ & {$[91]$} \\
\hline Aleksan a) & 0.08 & $64-128$ & $\mathrm{Pd} / \mathrm{Ti}$ & [92] \\
\hline Miskelly & $?$ & 64 & $\mathrm{Pd}$ & [93] \\
\hline Alber & 0.3 & 120 & $\mathrm{Pd}$ & [94] \\
\hline Blagus a) & 0.3 & $18-200$ & $\mathrm{Pd}$ & [95] \\
\hline Schilling & 0.4 & $3-4$ & $\mathrm{Pd}$ & [72] \\
\hline Baurichter & 30 & $20-80$ & $\mathrm{Pd}$ & [96] \\
\hline Schrieder & 3 & 11 & $\mathrm{Pd}$ & [97] \\
\hline Roberts & 7 & $50-250$ & $\mathrm{Pd}$ & [98] \\
\hline
\end{tabular}

a) The current was not varied during the runs.

b) There was only searched for excess heat. The upper limit was $10^{-12} \mathrm{~W} / \mathrm{cm}^{3}$.

Table 2

Deuteron implantation experiments.

\begin{tabular}{|c|c|c|c|c|c|}
\hline First author & $\begin{array}{l}\text { Sensitivity } \\
\left(10^{-24} \mathrm{~d}-\mathrm{d}^{-1} \mathrm{~s}^{-1}\right)\end{array}$ & $\begin{array}{l}\text { Current } \\
\left(\mathrm{mA} / \mathrm{cm}^{2}\right)\end{array}$ & $\begin{array}{l}\text { Voltage } \\
(1000 \mathrm{~V})\end{array}$ & Material & References \\
\hline Durocher & $?$ & 10 & 60 & $\mathrm{Pd} / \mathrm{In}$ & {$[99,100]$} \\
\hline Behrisch & 8 & 17 & 1.5 & $\mathbf{T i}$ & {$[101]$} \\
\hline Roth $^{c)}$ & 10 & 0.3 & $0.3-6$ & $\mathrm{Ti}$ & {$[102]$} \\
\hline Chambers & 350000 & 0.11 & 1.5 & $\mathrm{Pd}$ & [103] \\
\hline
\end{tabular}

\footnotetext{
c) The voltage was not varied during the runs.
} 
Table 3

Gas loaded experiments

\begin{tabular}{|c|c|c|c|c|}
\hline First author & $\begin{array}{l}\text { Sensitivity } \\
\left(10^{-24} \mathrm{~d}-\mathrm{d}^{-1} \mathrm{~s}^{-1}\right)\end{array}$ & $\begin{array}{l}\text { Temperature } \\
\text { (K) }\end{array}$ & Material & References \\
\hline McCracken & 36 & $77-293$ & $\mathrm{Ti}$ & [104] \\
\hline Price & $?$ & 293 & $\mathrm{Li}$ & {$[70]$} \\
\hline Price & 20 & $77-293$ & $\mathrm{Pd} / \mathrm{Ti}$ & [90] \\
\hline Botter & 0.002 & 293 & $\mathrm{Pd}$ & {$[105]$} \\
\hline Eberhard & 0.1 & $77-293$ & $\mathrm{Ti}$ & {$[106]$} \\
\hline Balke & 0.005 & $77-293$ & $\mathrm{Pd} / \mathrm{Ti}$ & {$[107]$} \\
\hline Silvera & $2.5 \times 10^{6}$ & $4.3-400$ & $\mathrm{Pd}$ & {$[108]$} \\
\hline
\end{tabular}

the signals at will, so the mean rate might be much smaller ).

(4) According to Menlove et al. [13] there occurs meanly one burst per $20 \mathrm{~h}$ at temperatures about 200 $\mathrm{K}$ and one burst per $400 \mathrm{~h}$ at room temperature (if there is used about $100 \mathrm{~g}$ of $\mathrm{Ti}$ ). Hence, when one looks for bursts one has to examine a number of temperature cycles, because the bursts occurred while the cells where warmed up. Price [70] might have detected no protons, because he had performed only about 100 cracks. Menlove et al. [13], however, have measured tens of thousands of acoustical emissions, where only six bursts have taken place. This means that less than one out of 1000 cracks yields bursts.

We see that apart from the experiments of Kashy et al. [71] and Schilling et al. [72] all the experiments listed in tables $1-3$ can be attributed to a minimum number of possible sources of failure above.

Eventually, the current applied by Kashy et al. [71] and Schilling et al. [72] has been too small, i.e. the variations of current might have been without action.

\section{References}

[1] S.E. Jones, E.P. Palmer, J.B. Czirr, D.L. Decker, G.L. Jensen, J.M. Thorne, S.F. Taylor and J. Rafelski, Nature 338 (1989) 737.

[2] S.E. Jones, D.L. Decker and H.D. Tolley. Nature 343 (1990) 703 .

[3] S.E. Jones, E.P. Palmer, J.B. Czirr, D.L. Decker, G.L. Jensen, J.M. Thorne, S.F. Taylor and J. Rafelski, J. Fusion Energy 9 (1990) 199.

[4] S.E. Jones, report Ettore Majorana Centre Conf., Erice, 12 April 1989

[5] S.E. Jones, report ACS-Meeting, Dallas, 19 April 1989.

[6] S.E. Jones, report APS-Meeting, Baltimore, 1 May 1989.
[7] S.E. Jones, report ECS-Meeting, Los Angeles, 8 May 1989.

[8] S.E. Jones, report LANL-Meeting, Santa Fe, 23-25 May 1989.

[9] S.E. Jones, J.B. Czirr, D.L. Decker, G.L. Jensen and E.P. Palmer, Response to queries regarding "Observation of cold nuclear fusion in condensed matter", 1989. unpublished.

[10] A. Bertin. M. Bruschi, M. Capponi, S. De Castro. U. Marconi, C. Moroni, M. Piccinini, N. Semprini-Cesari, A. Trombini, A. Vitale, A. Zoccoli, S.E. Jones, J.B. Czirr, G.L. Jensen and E.P. Palmer. Nuovo Cimento 101A (1989) 997.

[11] A. Bertin, report LANL-Meeting. Santa Fe, 23-25 May 1989.

[12] A. Bertin, M. Bruschi, M. Capponi, S. De Castro, U. Marconi, C. Moroni, M. Piccinini, N. Semprini-Cesari, A. Trombini, A. Vitale, A. Zoccoli, S.E. Jones, J.B. Czirr, G.L. Jensen and E.P. Palmer, on the criteria followed in the Gran Sasso measurement on cold nuclear fusion, 1989. unpublished.

[13] H.O. Menlove, M.M. Fowler, E. Garcia, M.C. Miller, M.A. Paciotti, R.R. Ryan and S.E. Jones, J. Fusion Energy 9 (1990) 495.

[14] H.O. Menlove, report LANL-Meeting, Santa Fe, 23-25 May 1989.

[15] S.E. Jones, report Oxford Conf. on Muon catalyzed and cold fusion, Oxford, September 1989.

[16] D.R.O. Morrison, report APS-Meeting, Baltimore, I May 1989.

[17] S. Freedman and D. Krakauer. Nature 343 (1990) 703.

[18] J.M. Carpenter. Nature 338 (1989) 711.

[19] M. Gai, S.L. Rugari, R.H. France, B.J. Lund, Z. Zhao, A.J. Davenport, H.S. Isaacs and K.G. Lynn, Nature 340 (1989) 29

[20] E.M. Attas. K.W. Chambers, W. Dueck, R. Dutton and A.K. McIlwain, Nature 344 (1990) 390.

[21] A.J. McEvoy and C.T.D. O'Sullivan. Nature 338 (1989) $711 ; 339$ (1989) 186 (E)

[22] R. Pool, Science 244 (1989) 284.

[23] R.W. Kühne, Phys. Lett. A 155 (1991) 467.

[24] R.W. Kühne, Observational evidence for microscopical hot fusion. Phys. Rev. C (1991), submitted. 
[25] J.S. Cohen and J.D. Davies, Nature 338 (1989) 705.

[26] J.S. Cohen and J.D. Davies, Nature 342 (1989) 488.

[27] T. Takeda and T. Takizuka, J. Phys. Soc. Japan 58 (1989) 3073.

[28] S.E. Segre, S. Atzeni, S. Briguglio and F. Romanelli, Europhys. Lett. 11 (1990) 201.

[29] S.E. Segre, S. Atzeni, S. Briguglio and F. Romanelli, report Nuovo Cimento Conf., Varenna, 15-16 September 1989

[30] V.A. Klyuev, A.G. Lipson, Yu.P. Toporov and B.V. Deryagin, Sov. Phys. Tech. Lett. 12 (1986) 551.

[31] A.G. Lipson, D.M. Sakov, V.A. Klyuev, B.V. Deryagin and Yu.P. Toporov, JETP Lett. 49 (1989) 675.

[32 ] B.V. Deryagin, A.G. Lipson, V.A. Klyuev, D.M. Sakov and Yu.P. Toporov, Nature 341 (1989) 392.

[33] R. Seitz, Nature 339 (1989) 185.

[34] V.I. Goldanskii and F.I. Dalidchik, Nature 34? (1989) 231.

[35] P.I. Golubnichii, V.A. Kurakin, A.D. Filonenko, V.A. Tsarev and A.A. Tsarik, Sov. Phys. Dokl. 34 (1989) 628.

[36] J.T. Dickinson, L.C. Jensen, S.C. Langford, R.R. Ryan and E. Garcia, J. Mater. Res. 5 (1990) 109.

[37] H. Craig, J.E. Lupton, J.A. Welhan and R. Poreda, Geophys. Res. Lett. 5 (1978) 897.

[38] J.E. Lupton and H. Craig, Science 214 (1981) 13.

[39] B.A. Mamyrin and L.N. Tolstikhin, Helium isotopes in nature (Elsevier, Amsterdam, 1984).

[40] B.A. Mamyrin, L.V. Khabarin and V.S. Yudenich, Sov. Phys. Dokl. 23 (1978) 581.

[41] C. DeW. Van Siclen and S.E. Jones, J. Phys. G 12 (1986) 213.

[42] S.E. Jones, A.N. Anderson, A.J. Caffrey, J.B. Walter, K.D. Watts, J.N. Bradbury, P.A.M. Gram, M. Leon, H.R. Maltrud and M.A. Paciotti, Phys. Rev. Lett. 51 (1983) 1757.

[43] S.E. Jones, in: Atomic physics 9, eds. R.S. Van Dyck and E.N. Fortson (World Scientific, Singapore, 1984) p. 99.

[44] S.E. Jones, Fusion Tech. 8 (1985) 1521.

[45] S.E. Jones, A.N. Anderson, A.J. Caffrey, C. DeW. Van Siclen, K.D. Watts, J.N. Bradbury, J.S. Cohen, P.A.M. Gram, M. Leon, H.R. Maltrud and M.A. Paciotti, Phys. Rev. Lett. 56 (1986) 588.

[46] S.E. Jones, Nature 321 (1986) 127.

[47] J. Rafelski, Bull. Am. Phys. Soc. 34 (1989) 1859.

[48] B. Delley, Europhys. Lett. 10 (1989) 347.

[49] F. Marchesoni, C. Presilla and F. Sacchetti, Europhys. Lett. 10 (1989) 493.

[50] F. Marchesoni, C. Presilla and F. Sacchetti, report Nuovo Cimento Conf., Varenna, 15-16 September 1989.

[51] R. Bailan, J.P. Blaizot and P. Bonche, J. Phys. (Paris) 50 (1989) 2307

[52] R.L. Garwin, Nature 338 (1989) 616.

[53] S.E. Koonin, report Ettore Majorana Centre Conf., Erice, 12 April 1989.

[54] S.E. Koonin, report APS-Meeting, Baltimore, I May 1989.

[55] S.E. Koonin, Bull. Am. Phys. Soc. 34 (1989) 1859

[56] S.E. Koonin and M. Nauenberg, Nature 339 (1989) 690.

[57] A.J. Leggett and G. Baym, Nature 340 (1989) 45.
[58] D. Lindley, Nature 344 (1990) 375.

[59] B.J.B. Crowley, Nucl. Fusion 29 (1989) 2199.

[60] N.R. Arista, A. Gras-Marti and R.A. Baragiola, Phys. Rev. A 40 (1989) 6873.

[61] X.W. Wang, S.G. Louie and M.L. Cohen, Phys. Rev. B 40 (1989) 5822.

[62 ] P.M. Richards, Phys. Rev. B 40 (1989) 7966.

[63] C.J. Benesh and J.P. Vary, Phys. Rev. C 40 (1989) 495.

[64] C.J. Horowitz, Phys. Rev. C 40 (1989) 1555

[65]Z. Sun and D. Tomanek, Phys. Rev. Lett. 63 (1989) 59

[66] A.J. Leggett and G. Baym, Phys. Rev. Lett. 63 (1989) 191.

[67] V.I. Goldanskii, and F.I. Dalidchik, Phys. Lett. B 234 (1990) 465.

[68] K. Langanke, H.J. Assenbaum and C. Rolfs, Z. Phys. A 333 (1989) 317.

[69] J. Kondo, J. Phys. Soc. Japan 58 (1989) 1869.

[70] P.B. Price, Nature 343 ( 1990 ) 542.

[71 ] E. Kashy, W. Bauer, Y. Chen, A. Galonsky, J. Gaudiello, M. Maier, D.J. Morrissey, R.A. Pelak, M.B. Tsang and J. Yurkon, Phys. Rev. C 40 (1989) 1.

[72] K.D. Schilling, P. Gippner, W. Seidel, F. Stary and D. Wohlfarth, Z. Phys. A 336 (1990) 1.

[73] R.D. Armstrong, E.A. Charles, I. Fells, L. Molyneux and M. Todd, Electrochim. Acta 34 (1989) 1319.

[74] R.D. Armstrong, E.A. Charles, I. Fells, L. Molyneux and M. Todd, J. Elec. Chem. 272 (1989) 293.

[75] M. Gai, report LANL-Meeting, Santa Fe, 23-25 May 1989.

[76] N.S. Lewis, C.A. Barnes, M.J. Heben, A. Kumar, S.R. Lunt, G.E. McManis, G.M. Miskelly, P.M. Penner, M.J. Sailor, P.G. Santangelo, G.A. Shreve, B.J. Tufts, M.G. Youngquist, R.W. Kavanagh, S.E. Kellogg, R.B. Vogelaar, T.R. Wang, R. Kondrat and R. New, Nature 340 (1989) 525 .

[77] N.S. Lewis, report APS-Meeting, Baltimore, 1 May 1989.

[78] N.S. Lewis, report ECS-Meeting, Los Angeles, 8 May 1989.

[79] D.E. Williams, D.J.S. Findlay, D.H. Craston, M.R. Sené, M. Bailey, S. Croft, B.W. Hooton, C.P. Jones, A.R.J. Kucernak, J.A. Mason and R.I. Taylor, Nature 342 (1989) 375.

[80] D.E. Williams, D.J.S. Findlay, D.H. Craston, M.R. Sené, M. Bailey, S. Croft, B.W. Hooton, C.P. Jones, A.R.J. Kucernak, J.A. Mason and R.I. Taylor, report Nuovo Cimento Conf., Varenna, 15-16 September 1989.

[81] M.H. Salamon, M.E. Wrenn, H.E. Bergeson, K.C. Crawford, W.H. Delaney, C.L. Henderson, Y.Q. Li, J.A Rusho, G.M. Sandquist and S.M. Seltzer, Nature 344 (1990) 401 .

[82] M.H. Salamon, report NSF/EPRI-Meeting, Washington, 16-18 October 1989.

[83] M.M. Broer, L.C. Feldman, A.C.W.P. James, J.S. Kraus and R.S. Raghavan, Phys. Rev. C 40 (1989) 1559.

[84] M.M. Broer, report Ettore Majorana Centre Conf., Erice, 12 April 1989.

[85] M.R. Deakin, J.D. Fox, K.W. Kemper, E.G. Myers, W.N. Shelton and J.G. Skofronick, Phys. Rev. C. 40 (1989) 1851 . 
[86] K.E. Rehm, W. Kutschera and G.J. Perlow, Phys. Rev. C $41(1990) 45$.

[87] J.R. Southon, J.W. Stark, J.S. Vogel and J.C. Waddington, Phys. Rev. C 41 (1990) 1899.

[88] J.F. Ziegler, T.H. Zabel, J.J. Cuomo, V.A. Brusic, G.S. Cargill, E.J. O'Sullivan and A.D. Marwick, Phys. Rev. Lett. 62 (1989) 2929

[89] J.F. Ziegler, report Ettoro Majorana Centre Conf., Erice, 12 April 1989.

[90] P.B. Price, S.W. Barwick. W.T. Williams and J.D. Porter. Phys. Rev. Lett. 63 (1989) 1926.

[91] J.P. Briand, G. Ban, M. Froment, M. Keddam and F. Abel, Phys. Lett. A 145 (1990) 187.

[92] R. Aleksan. M. Avenier, G. Bagieu, J. Bouchez, J.F. Cavaignac, J. Collot, M.C. Cousinou, Y. Declais, Y. Dufour, R. Durand, R. Faure, J. Favier, E. Kajfasz, H. De Kerret, D.H. Koang, B. Lefièvre, E. Lesquoy, J. Mallet, E. Nagy, M. Obolensky, H. Pessard, F. Pierre, A. Stutz and J.P. Wuthrick, Phys. Lett. B 234 (1990) 389.

[93] G.M. Miskelly, M.J. Heben, A. Kumar, R.M. Penner, M.J. Sailor and N.S. Lewis, Science 246 (1989) 793.

[94] D. Alber, O. Boebel, C. Schwarz, H. Duwe, D. Hilscher, H. Homeyer, U. Jahnke and B. Spellmeyer, Z. Phys. A 333 (1989) 319.

[95] S. Blagus, M. Bogovac, D. Hodko, M. Krcmar, D. Miljanic, P. Tomas, M. Vajić and M. Vuković, Z. Phys. A 333 (1989) 321.

[96] A. Baurichter, W. Eyrich, M. Frank, H. Göhr, W. Kreische, H. Ortner, B. Röseler, C.A. Schiller, G. Weeske and W. Witthun, Z. Phys. B 76 (1989) 1.
[97] G. Schrieder, H. Wipf and A. Richer. Z. Phys. B 76 (1989) 141.

[98 ] D.A. Roberts, F.D. Becchetti, E. Ben-Jacob, P. Garik, J. Musser, B. Orr, G. Tarlé, A. Tomasch, J.S. Holder, D. Redina, B. Heuser and G. Wicker, Phys. Rev. C 42 (1990) 1809.

[99] J.J.G. Durocher, D.M. Gallop, C.B. Kwok, M.S. Mathur, J.K. Mayer, J.S.C. McKee, A. Mirzai, G.R. Smith, Y.H. Yeo, K.S. Sharma and G. Williams, Can. J. Phys. 67 (1989) 624.

[100] J.S.C. McKee, report APS-Meeting, Baltimore, I May 1989.

[101] R. Behrisch, W. Möller, J. Roth and B.M.U. Scherzer. Nucl. Fusion 29 (1989) 1187.

[ 102 ] J. Roth, R. Behrisch, W. Möller and W. Ottenberger, Nucl. Fusion 30 (1990) 441.

[103] G.P. Chambers, J.M. Eridon and K.S. Grabowski, Phys. Rev. B 41 (1990) 5388.

[104] G.M. McCracken, M. Bailey, S. Croft, D.J.S. Findlay, A Gibson, R.P. Govier, O.N. Jarvis, H.J. Milton, B.A. Powell, G. Sadler, M.R. Sené, D.R. Sweetman, P. van Belle and H.H.H. Watson, J. Phys. D 23 (1990) 469.

[105] F. Botter, J. Bouchez, J. Collot, E. Kajfasz, B. Lefièvre, E. Lesquoy, A. Stutz, S. Tistchencko and S. Zylberajch, Phys. Lett. B 232 (1989) 536.

[106] V. Eberhard, W. Heeringa, H.O. Klages, R. Maschuw, G. Völker and B. Zeitnitz, Z. Phys. A 334 (1989) 357.

[ 107 ] B. Balke, L. Cox. O. Fackler, M. Mugge, P.C. Souers, R.T Tsugawa and R.M. White, Phys. Rev. C. 42 (1990) 30.

[ 108 ] I.F. Silvera and F. Moshary. Phys. Rev. B 42 (1990) 9143. 